\title{
Referral criteria for school dental screening
}

The development of a consensus among primary care dentists of referral criteria for school dental screening P. I. Kearney-Mitchell, K. M. Milsom, A. S. Blinkhorn and M. Tickle Br Dent J 2006; 200: 509-512

\section{Objective}

To obtain consensus amongst a sample of primary care dentists in the North West of England on a set of clinical criteria that should trigger referral following school dental screening.

Design

Delphi process.

Setting

Primary dental care, England 2002.

Method

Primary care dentists in the North West of England were randomly selected to complete a two round 'Delphi exercise' that included 10 potential referral criteria. The dentists were invited to express their level of support for the inclusion of each referral criterion.

Main outcome measures

Level of agreement for each referral criterion. Acceptance of any criterion was that the interquartile range should be no more than 3 scale points with the lower value being no less than 7 .

Results

Eighty-eight dentists, (72.7\%), completed the Delphi exercise. Six referral criteria met with the groups' approval:

- Child with caries in permanent dentition

- Child with darkened/discoloured permanent incisors

- Child aged 9-10 years with overjet greater than $10 \mathrm{~mm}$

- Child over six years with either gross plaque, calculus or swollen gums

- Child with evidence of sepsis

- Child registered with a GDP with caries in permanent dentition.

Conclusion

It is possible for a representative sample of primary care dentists in the North West to agree referral criteria following school dental screening.

\section{IN BRIEF}

- It is possible for primary care dentists to agree referral criteria following school dental screening.

- This study reinforces earlier research which suggests that caries in the primary dentition is not perceived as an important trigger for referral following school dental screening.

- Standardisation of the referral criteria used in school dental screening is likely to enhance the quality of this dental public health activity.

- School dental screening is not seen by the profession as a vehicle to increase dental registration.

\section{COMMENT}

Current legislation in England and Wales demands that each child receives a dental screening at least three times during their school years. ${ }^{1}$ There is a considerable financial cost associated with fulfilling this requirement. ${ }^{2}$ Furthermore, there is an opportunity cost as the resources consumed by school dental screening are not available for other primary dental care priorities. It is therefore important that school dental screening programmes are as effective and efficient as possible.

In the UK the child dental screening system is split. The Community Dental Service generally undertakes the screening exercise while the General Dental Service usually carries out any subsequent treatment. If treatment philosophies differ between these services locally, the cost-effectiveness of the screening programme may be reduced and parents' confidence in the GDS and/or the CDS undermined. The methodology used in this study to agree referral criteria is practical and it could be adopted in other regions.

There has been extensive debate in the pages of this journal on the value of restoring carious deciduous teeth. Regardless of the merits and demerits on either side of the argument, it is clear from this study that in the North West of England primary care dentists do not view a child with carious deciduous teeth as requiring referral following screening. Whether this is because they believe that treatment is not required or that available treatments are ineffective is not clear. Nevertheless, in pure screening terms one could argue that these children are 'at risk' and so referral would be appropriate for preventive interventions. Of course, the remuneration system needs to be in harmony with this rationale and adequately reward prevention.

Similarly, while the benefits of dental registration or otherwise have been the subject of much discussion, the primary care dentists involved in this study do not seem to feel that increasing registration levels is in itself a worthwhile exercise. If the views of this sample are representative of primary care dentists across the UK it goes some way to explaining why initiatives designed to increase dental registration have enjoyed only limited success.

As the debate on the value of school dental screening programmes once again ignites it is with eager anticipation that we await the findings from this group's wider study on the effectiveness of this public health activity.

M. Donaldson, Consultant in Dental Public Health, Northern Health and Social Services Board, Northern Ireland

1. Department of Health. HSG(97)4: Primary Care Dental Services. London: HMSO, 1997

2. Milsom K. School dental screening - what value? Br Dent J 1995; 178: 322.

doi: $10.1038 /$ sj.bdj.4813526 